\title{
MOLECULAR VIBRATION APPROACH TO POLARIZABILITIES OF METHYL CINNAMATE LIQUID CRYSTAL COMPOUNDS
}

\author{
Y.N. MURTHY \\ Department of Physics, SSBN College, Anantapur-515 001 (A.P.), India \\ V.R. Murthy and R.N.V. Ranga Reddy \\ Department of Physics, S.K. University, Anantapur-515 003 (A.P.), India \\ (Received July 1, 1996; revised version December 16, 1996; \\ in final form March 12, 1997)
}

A new theoretical method of evaluating polarizabilities of liquid crystals is presented and applied to the liquid crystalline compounds, namely $n$-alkyl-p-(4-ethoxy benzylidene amino)- $\alpha$-methyl cinnamates, which exhibit smectic $A$ and nematic phases. In the present method, vibrational frequencies are used to evaluate force constants, mean amplitude of vibration and hence bond polarizabilities. From mean polarizabilities, polarizability anisotropies and mean diamagnetic susceptibilities are also estimated. A close agreement is found between the values estimated from the present molecular vibration method and the reported data, which confirms the applicability of this method to the liquid crystals exhibiting smectic $A$ and nematic phases. In addition, the variation of the order parameter with temperature is also studied.

PACS numbers: $61.30 .-\mathrm{v}$

\section{Introduction}

Molecular spectroscopic studies have gained much interest in recent years to study the structure and physical properties of liquid crystals. These studies are quite helpful in getting a better understanding of the molecular and structural characteristics of mesogenic materials. The type of mesophases and their thermal and physical characteristics are strongly dependent on the steriochemistry and the structure of molecules. Some substituents in lateral or terminal position may affect liquid crystallinity in a number of ways, since many of the physical properties, length, breadth, polarizability, coplanarity, dipole moments etc., get varied simultaneously. 
The molecular polarizabilities and their anisotropy are important parameters in characterizing liquid crystalline substances, since they play a significant role in the intermolecular interactions. Molecular polarizabilities, though impossible to measure directly can be calculated on the basis of different models. There are different methods of evaluating mean molecular polarizabilities. Among them the refractivity method, the magneto-optic method of Faraday, the method based on the modified Lippin cot- $\delta$-potential model and the molecular vibration method are a few ones. The first two methods are purely experimental and the last two are theoretical. The refractivity method is useful, if the system under study is either transparent or approachable by solution techniques. The magneto-optic method requires relatively expensive instrumentation. In cases when the best instrumentation is not available readily, the last two theoretical methods can be used. In the two theoretical methods, the molecular vibration method is superior to the other since any small deviation in intermolecular forces due to changes in chemical environment will be truly represented in their force constants and vibrational frequencies. The changes in vibrational frequencies in cis and trans configurations and ortho, para and meta substitutions are examples. Therefore, the molecular vibration method can be treated as superior to Lippin $\cot -\delta$-potential model which requires only bond lengths and atomic delta functions and reduced electronegativities. Both bond lengths and reduced electronegativities are less sensitive to conformational changes than vibrational frequencies.

In the present investigation the molecular polarizabilities, the polarizability anisotropies, the diamagnetic susceptibilities and the order parameters of the homologous series of $n$-alkyl-p-(4-ethoxy benzylidene amino)- $\alpha$-methyl cinnamates are evaluated. These compounds have the chemical formula

$$
\mathrm{C}_{2} \mathrm{H}_{5} \mathrm{O}\left(\mathrm{C}_{6} \mathrm{II}_{4}\right) \mathrm{CHI}: \mathrm{N}\left(\mathrm{C}_{6} \mathrm{II}_{4}\right) \mathrm{CII}: \mathrm{C}\left(\mathrm{CII}_{3}\right)(\mathrm{COOR})
$$

where $\mathrm{R}$ is the alkyl group $\mathrm{CH}_{3}-\left(\mathrm{CII}_{2}\right) n$ with $n=1$ to 8 . The transition temperatures of these compounds are given below (Table I).

TABLE I

Transition temperatures of $n$-alkyl-p-(4-ethoxy benzylidene amino)$\alpha$-methyl cinnamates.

\begin{tabular}{c|c|c|c}
\hline \hline $\mathrm{R}$ & Smectic $A\left[{ }^{\circ} \mathrm{C}\right]$ & Nematic $\left[{ }^{\circ} \mathrm{C}\right]$ & Isotropic $\left[{ }^{\circ} \mathrm{C}\right]$ \\
\hline Ethyl ester $(n=1)$ & 77.0 & 94.0 & 123.0 \\
Propyl ester $(n=2)$ & 81.9 & 90.9 & 121.8 \\
Butyl ester $(n=3)$ & 59.4 & 86.0 & 100.6 \\
Amyl ester $(n=4)$ & 65.4 & 84.2 & 100.5 \\
Hexyl ester $(n=5)$ & 88.6 & 83.0 & 91.2 \\
Heptyl ester $(n=6)$ & 62.0 & 82.3 & 90.2 \\
Octyl ester $(n=7)$ & 57.0 & 81.8 & 85.3 \\
Nonyl ester $(n=8)$ & 58.8 & 82.4 & 85.1
\end{tabular}




\section{Theory}

The details of the present approach are briefly given below. The polarizability of a molecule is associated with its vibration. When a molecule is displaced from its equilibrium position, the equilibrium is restored by restoring force, which per unit displacement was found to be proportional to the square of the frequency of vibration. Thus one can expect a relation between polarizability $(\alpha)$ and the force constant $(K)$. One such relation was proposed by Fripiat [1] which reads as

$$
K=A+\frac{B}{\alpha_{0}} .
$$

$A$ and $B$ are constants for a given series of compounds. $A$ depends on the nature of the bond and represents force designated as "quantique". $B / \alpha_{0}$ represents the contribution from the electronic deformability of ions.

Lefevre and Narayana Rao [2] proposed a general method of computing atomic polarizabilities from stretching $(K)$ and bending $\left(K_{\mathrm{s}}\right)$ force constants. Their relation for $X Y_{2}$ bent symmetric type molecule with $Y X Y=2 \theta$ is

$$
A^{P}=\frac{4 \pi N \mu^{2} \sin ^{2} \theta}{9 K_{\delta}}+\frac{8 \pi N e^{2} \cos ^{2} \theta}{9 K}+\frac{8 \pi N e^{2} \sin ^{2} \theta p^{2}}{9 K r^{2}},
$$

where $\mu$ is the bond moment, $K_{\delta}$ is the bending force constant, $A^{P}$ is the atomic polarizability and $N$ is the bond order.

In an attempt to develop a more general method, Rao and Murthy [3] have developed an equation relating longitudinal $\left(b_{\mathrm{L}}\right)$ and transversal $\left(b_{\mathrm{T}}\right)$ bond polarizabilities with the stretching force constant $(K)$. They also proposed another empirical relation between the polarizability and mean amplitude of vibration. These two relations are given below.

\subsection{Relation 1}

Lefevre et al. [4] have attributed the observed molar Kerr constant and depolarization factor in spherically symmetric molecules (e.g. methane) to the deviations in sphericity which arises out of the distortions in bond angles brought in by the external electric field. The obscrved electric birefringence and hence molar Kerr constant have been accounted for by a temperature independent term 0 as

where

$$
\theta=\frac{8}{5}\left(B_{1}+C\right)
$$

$$
B_{1}=\frac{\mu^{2}}{18 K_{\delta}^{2}}\left(b_{\mathrm{L}}-b_{\mathrm{T}}\right)
$$

and

$$
C=\frac{2 \mu e}{9 K K_{\delta}}\left(\frac{\mathrm{d} b_{\mathrm{L}}}{\mathrm{d} r}-\frac{\mathrm{d} b_{\mathrm{T}}}{\mathrm{d} r}\right) .
$$

IIere $\mu$ is the bond moment, $e$ is the effective charge and $\mathrm{d} b_{\mathrm{L}} / \mathrm{d} r, \mathrm{~d} b_{\mathrm{T}} / \mathrm{d} r$ are longitudinal and transversal bond polarizability derivatives with respect to the internuclear separation $(r)$ respectively. 
Rearranging Eq. (5) we get

$$
\mathrm{d}\left(b_{\mathrm{L}}-b_{\mathrm{T}}\right)=\left(9 K_{\delta} C / 2 \mu e\right) K \mathrm{~d} r .
$$

According to Gordy [5] the relation between $K$ and $r$ is

$$
K=\left(\frac{X_{1} X_{2}}{r^{2}}\right)^{3 / 4} a N+b,
$$

where $X_{1}, X_{2}$ are the electronegativities of atoms forming the bond of length of $r$ and $N$ is the bond order. $a$ and $b$ are Gordy's [5] constants. Substituting (7) in (6) and integrating

$$
b_{\mathrm{L}}-b_{\mathrm{T}}=\frac{9 K_{\delta} C}{2 \mu e}(3 b-2 k) r+P
$$

$P$ is an integration constant. As $r \rightarrow 0, b_{\mathrm{L}}-b_{\mathrm{T}}$ vanishes much faster than the $b_{\mathrm{L}}$ and $b_{\mathrm{T}}$ values themselves and hence $P=0$.

On further integrating $\mathrm{Eq}$. (8) and simplifying

$$
b_{\mathrm{L}}-b_{\mathrm{T}}=A\left[\left(X_{1} X_{2}\right)^{1 / 2}\left(\frac{a N}{K-b}\right)^{2 / 3}\right]^{S},
$$

where $S=K /(3 b-2 k)$. The parameter $A$ is characteristic of the bond under consideration and is given by

$$
A=0.1\left(\frac{(i j)_{y}}{(i j)_{x}}\right)
$$

where $(i j)$ refers to the product of row and column numbers of an atom in the periodic table.

\subsection{Relation 2}

Another empirical relation between mean polarizability of a bond and its mean amplitude of vibration is [6]

$$
b_{\mathrm{L}}+2 b_{\mathrm{T}}=C P^{j} j^{n r} \sigma^{1 / 2} .
$$

$C$ is a constant equal to $5.24 \times 10^{-15} . P$ is characteristic of the atom and is equal to $1,1.2,1.3,1.4$ and 1.5 according as it belongs to $2 \mathrm{nd}, 3 \mathrm{rd}, 4$ th, 5 th or 6 th row in the periodic table, $n$ is +1 or -1 according as the bond is nonhydride or hydride. $r$ is saturation factor.

From (9) and (11) it is now possible to evaluate longitudinal $\left(b_{\mathrm{L}}\right)$ and transversal $\left(b_{\mathrm{T}}\right)$ bond polarizabilities of each bond in the molecule. Then the average polarizability of the molecule is given by

$$
\alpha_{\mathrm{M}}=\frac{\sum n_{i}\left(b_{\mathrm{L}}+2 b_{\mathrm{T}}\right)_{i}}{3}
$$

where $n_{i}$ is the number of bonds of the type $i$. Murthy et al. [7], Subbaiah et al. [8-10] have used this method to evaluate $b_{\mathrm{L}}, b_{\mathrm{T}}$ and $\alpha_{\mathrm{M}}$ of few bio and synthetic polymers. 


\subsection{Estimation of molecular polarizability anisotropy}

It is a known fact that we need a molecular polarizability ellipsoid rather than the average polarizability to study the directional properties of the anisotropic crystals. In the calculation of the polarizability $\alpha_{\|}$, the molecular axis is taken along the line joining the centres of the two benzene rings and the molecule is assumed to be rigid. The polarizability contribution parallel to the molecular axis of the polarizability ellipsoid of a polyatomic molecule is given by

$$
\alpha_{\|}=\sum \bar{\alpha}_{\|} \cos ^{2} \theta+\sum \bar{\alpha}_{\perp} \sin ^{2} \theta
$$

where $\theta$ is the angle between a bond and the molecular axis, $\bar{\alpha}_{\|}$and $\bar{\alpha}_{\perp}$ are the parallel and perpendicular bond components of the polarizability, respectively.

In the absence of the confirmed molecular structures of many of the substances, for the sake of uniformity and simplicity the bond angles are assumed to be $109^{\circ}$, for the bonds involving tetrahedral linkage and in all other cases as $120^{\circ}$. Standard values of bond lengths are assumed and the angle between the various bonds and the molecular axis were determined by drawing the figure of the molecule.

Using the value of $\alpha_{\|}$obtained from Eq. (13) the molecular polarizability anisotropy can be written as

$$
\left(\alpha_{\|}-\alpha_{\perp}\right)=\frac{3}{2}\left(\alpha_{\|}-\bar{\alpha}\right)
$$

where $\bar{\alpha}$ is the mean polarizability obtained from molecular vibration method. $\alpha_{\perp}$ can be written as

$$
\alpha_{\perp}=\bar{\alpha}-\frac{1}{3}\left(\alpha_{\|}-\alpha_{\perp}\right)
$$

From the polarizability anisotropy $\left(\alpha_{\|}-\alpha_{\perp}\right)$, the scaling factor $\alpha /\left(\alpha_{\|}-\alpha_{\perp}\right)$ can also be estimated.

\subsection{Estimation of mean diamagnetic susceptibility}

The relation between diamagnetic susceptibility and mean polarizability is given as [11]

$$
-\chi=\left(\gamma m \sigma^{\prime}\right) \bar{\alpha},
$$

where $\gamma=(0.9)^{n}$ gives the saturation state of the molecule with $n$ denoting the number of unsaturated bonds and rings in the molecule; and $\sigma^{\prime}$ is the degree of covalency of the characteristic group and is given as

$$
\sigma^{\prime}=\left[\sigma_{1}^{1 / n 1} \cdot \sigma_{2}^{1 / n 2} \ldots \sigma_{p}^{1 / n p}\right]^{1 / 2},
$$

where $\sigma_{1}, \sigma_{2} \ldots \sigma_{p}$ are Pauling's [12] percentages of covalence characters of the bonds present in the characteristic group; $n 1, n 2 \ldots n p$ are the bond orders of the various bonds in the characteristic group, $m$ is a constant which is equal to $0.72 \times 10^{19}$. The value of $\sigma_{1}, \sigma_{2} \ldots \sigma_{p}$ are taken from Pauling [12]. 


\subsection{Estimation of order parameter}

The orientational order parameter $S$ is defined as [13]

$$
S=(1 / 2)\left\langle\left(3 \cos ^{2} \theta-1\right)\right\rangle,
$$

where $\theta$ is the angle made by the long molecular axis with the preferred direction and the brackets denote an average over the molecules in a microscopic volume.

The principal polarizabilities of the molecules, the anisotropic nature of the polarization field in the medium and the orientational order parameter $S$, determine the birefringence of nematic liquid crystals. For calculations of the polarizabilities of the molecules and of the order parameter $(S)$ from birefringence data, Lorentz-Lorentz formula cannot be employed because its validity is restricted to cases where there is spherical (or cubic) symmetry in connection with the molecular arrangement. According to Vuks [14] the ratio of the average local electric field to the applied field is the same for the two cases when the applied field is parallel and perpendicular to the optic axis of the medium. Neugebauer [15] has considered in detail the forms of the polarization field in anisotropic media. In a nematic medium, as a result of the anisotropic distribution of the molecules, the average local electric field is of the form $E+\gamma p$, where $E$ is the applied field, $p$ - the polarization and $\gamma$ is a factor which is different from directions parallel and perpendicular to the optic axis.

In the present investigation the order parameters of the homologous series of $n$-alkyl-p-(4-ethoxy benzylidene amino)- $\alpha$-methyl cinnamates, were evaluated by using isotropic internal field model of Vuks [14]. In the Vuks method the order parameter is given by

$$
S=\frac{\alpha}{\left(\alpha_{\|}-\alpha_{\perp}\right)} \frac{n_{\mathrm{e}}^{2}-n_{\mathrm{o}}^{2}}{n^{2}-1},
$$

where $n^{2}=\left(n_{\mathrm{e}}^{2}+2 n_{\mathrm{o}}^{2}\right) / 3$. Here $\alpha$ is the mean polarizability, $\alpha_{\|}$and $\alpha_{\perp}$ are the principal polarizabilities in direction parallel and perpendicular to the optic axis, respectively.

\section{Results and discussion}

The necessary data on vibrational frequencies of different bonds are taken from Landolt-Börnstein [16] and the necessary refractive indices are taken from the paper of Pelzl et al. [17].

The mean polarizabilities and polarizability anisotropies of the above homologous series of liquid crystals are presented in Tables II and III along with the reported values [18]. The mean diamagnetic susceptibilities are given in Table IV along with the values determined by Pascal [19] and Pacault [20] method and the Haberditzl [21, 22] method. A review of the data given in Table II shows that the polarizabilities, determined from molecular vibration method are in reasonably good agreement with those values obtained from modified Lippin cot- $\delta$-potential model method [23] as well as with the reported data [18]. This indicates the validity of the molecular vibration approach in evaluating the mean polarizability of liquid crystals. The mean diamagnetic susceptibilities obtained from molecular 
TABLE II

Polarizabilities $\left(\times 10^{24} \mathrm{~cm}^{3}\right)$ of $n$-alkyl-p-(4-ethoxy benzylidene amino)- $\alpha$-methyl cinnamates.

\begin{tabular}{c|c|c|c|c|c}
\hline \hline$n$ & $\sum_{i} b_{\mathrm{L} i}$ & $\sum_{i} b_{\mathrm{T} i}$ & \multicolumn{3}{|c}{ Mean molecular polarizability } \\
\cline { 4 - 6 } & & & $\begin{array}{c}\text { Present } \\
\text { method }\end{array}$ & $\begin{array}{c}\text { ML } \delta \mathrm{P}[23] \\
\text { method }\end{array}$ & $\begin{array}{c}\text { Reported [18] } \\
\text { values }\end{array}$ \\
\hline 1 & 59.99 & 34.40 & 42.93 & 43.42 & 43.00 \\
2 & 63.78 & 36.09 & 45.32 & 45.41 & 44.90 \\
3 & 67.56 & 37.78 & 47.71 & 47.10 & 46.70 \\
4 & 71.35 & 38.47 & 49.43 & 48.96 & 48.60 \\
5 & 75.14 & 40.16 & 51.82 & 50.62 & 50.40 \\
6 & 78.03 & 41.85 & 53.91 & 52.21 & 52.30 \\
7 & 81.72 & 43.54 & 56.27 & 53.81 & 54.10 \\
8 & 84.51 & 44.23 & 57.66 & 55.11 & 55.90 \\
\hline \multicolumn{3}{c}{$b_{\mathrm{L}}$ - longitudinal bond polarizability, $b_{\mathrm{T}}-$ transversal } \\
bond polarizability.
\end{tabular}

TABLE III

Polarizability anisotropy $\left(\times 10^{24} \mathrm{~cm}^{3}\right)$ of $n$-alkyl-p-(4-ethoxy benzylidene amino)- $\alpha$-methyl cinnamates.

\begin{tabular}{c|c|c|c|c|c}
\hline \hline$n$ & $\alpha_{1}$ & $\alpha_{\mathrm{t}}$ & $\Delta \alpha=\alpha_{1}-\alpha_{\mathrm{t}}$ & \multicolumn{2}{|c}{$\Delta \alpha / \alpha$} \\
\cline { 5 - 6 } & & & & $\begin{array}{c}\text { Present } \\
\text { method }\end{array}$ & $\begin{array}{c}\text { Reported } \\
\text { values }\end{array}$ \\
\hline 1 & 61.24 & 36.72 & 24.52 & 0.57 & 0.58 \\
2 & 64.89 & 39.66 & 25.23 & 0.55 & 0.56 \\
3 & 68.54 & 43.15 & 25.39 & 0.53 & 0.54 \\
4 & 72.19 & 46.98 & 25.21 & 0.50 & 0.52 \\
5 & 75.84 & 50.78 & 25.06 & 0.48 & 0.49 \\
6 & 79.49 & 54.58 & 24.91 & 0.45 & 0.47 \\
7 & 83.14 & 58.38 & 24.76 & 0.43 & 0.44 \\
8 & 86.79 & 62.26 & 24.53 & 0.41 & 0.42 \\
\hline
\end{tabular}

$\alpha_{1}$ - polarizability along the length of the axis, $\alpha_{\mathrm{t}}$ - polarizability in a direction perpendicular to the axis.

vibration approach also agree well with the results obtained from Pascal's and Haberditzl's methods.

The variation of mean polarizability and polarizability anisotropy with number of carbon atoms in alkyl chain is shown in Fig. 1. With increase in $n$, the mean polarizability increases while the polarizability anisotropy normalized against the 


\section{TABLE IV}

Mean diamagnetic susceptibilities $\left(\times 10^{5} \quad\right.$ CGS units $)$ of $n$-alkyl$p$-(4-ethoxy benzylidene amino)$\alpha$-methyl cinnamates.

\begin{tabular}{c|c|c|c}
\hline \hline \multirow{3}{*}{$n$} & \multicolumn{3}{|c}{ Mean diamagnetic susceptibility } \\
\cline { 2 - 4 } & $\begin{array}{c}\text { Present } \\
\text { method }\end{array}$ & $\begin{array}{c}\text { Pascal's } \\
\text { method }\end{array}$ & $\begin{array}{c}\text { Iaberditzl's } \\
\text { method }\end{array}$ \\
\hline 1 & 18.43 & 18.56 & 19.20 \\
2 & 19.51 & 19.60 & 20.21 \\
3 & 20.49 & 20.51 & 21.20 \\
4 & 21.60 & 21.58 & 22.12 \\
5 & 22.61 & 22.54 & 23.21 \\
6 & 23.52 & 23.68 & 24.25 \\
7 & 24.53 & 24.92 & 25.12 \\
8 & 25.54 & 25.93 & 26.27 \\
\end{tabular}

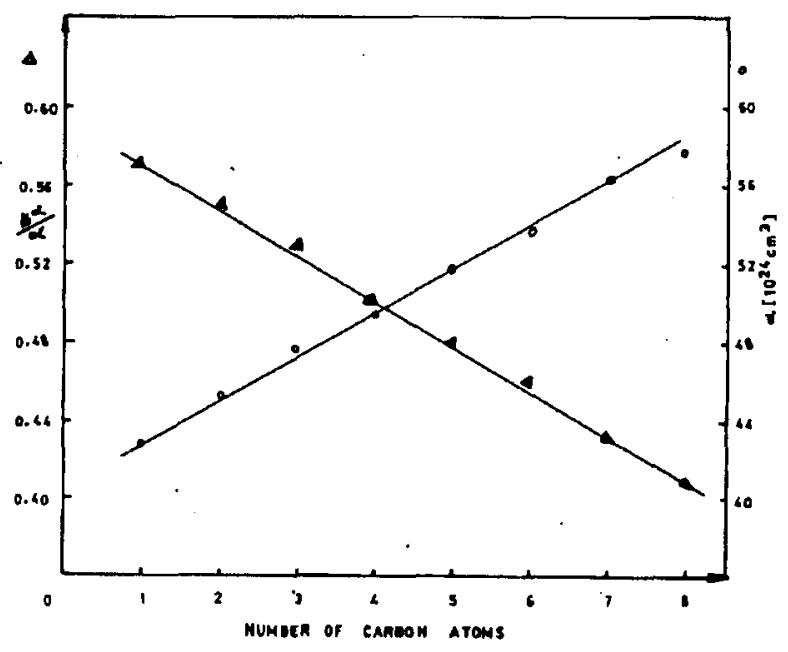

Fig. 1. Variation of mean polarizability and polarizability anisotropy with number of carbon atoms $(n)$.

a verage polarizability decreases almost linearly with the number of carbon atoms in the alkyl chain or with the increase in chain length. This suggests that the polarizability anisotropy mainly comes from the core contribution. The covalent bond electrons in the alkyl chain, on the other hand contribute more to the average polarizability than polarizability anisotropy. As a result, the addition of the $\mathrm{CH}_{2}$ groups to the alkyl chain increases $\alpha$ much more than $\Delta \alpha$, and so $\Delta \alpha / \alpha$ decreases with $n$. As $n$ increases from 1 to 8 , the mean polarizability $\alpha$ increases 


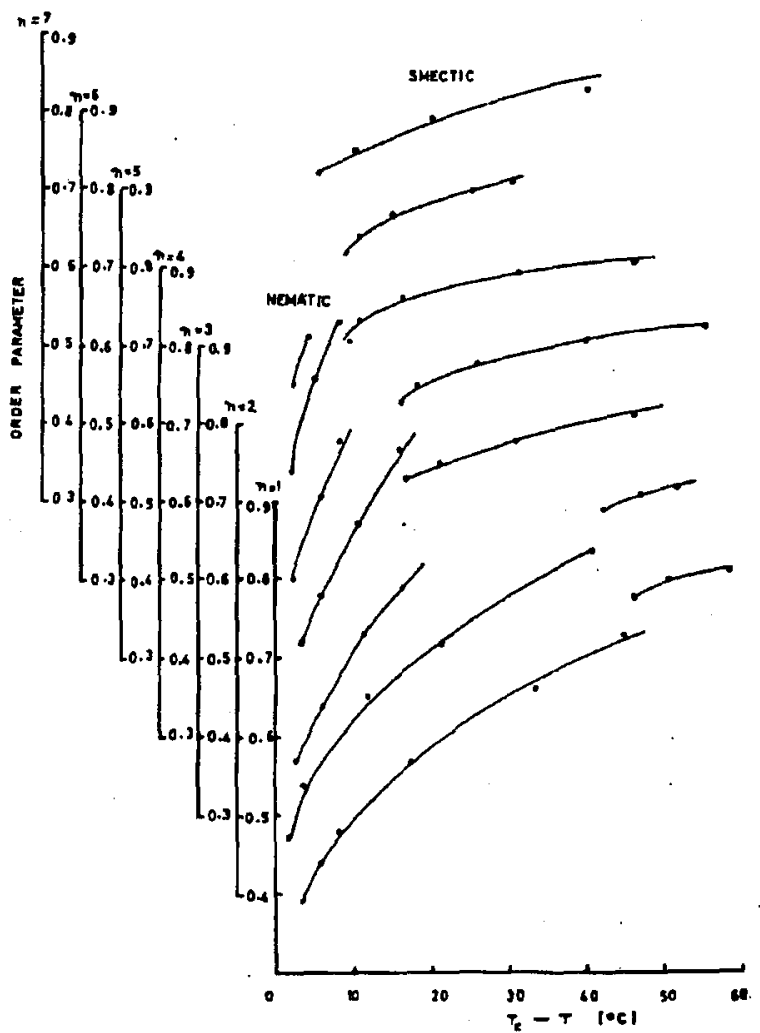

Fig. 2. Variation of order parameter with temperature.

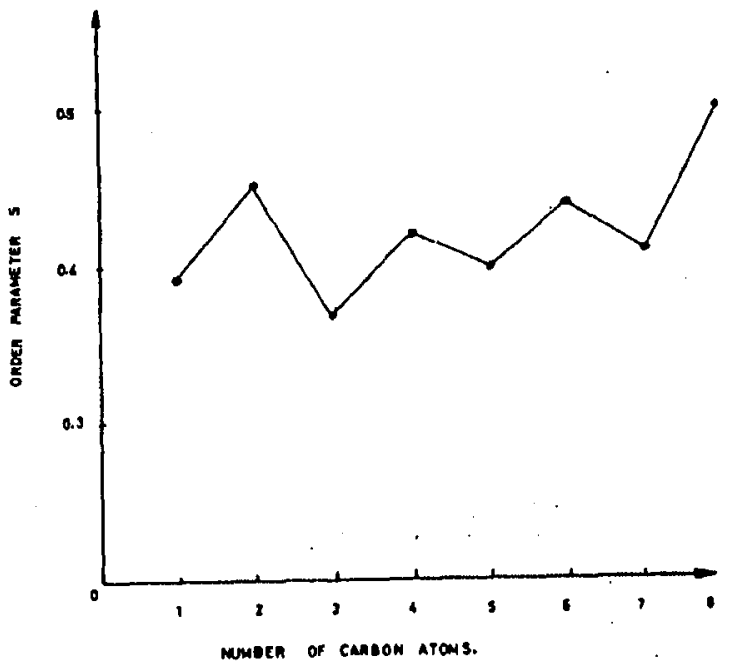

Fig. 3. Variation of order parameter with number of carbon atoms $(n)$. 
almost linearly by a factor of 1.34 while $\Delta \alpha$ shows a saturable increase in a factor of nearly 1 . The saturation of $\Delta \alpha$ at larger $n$ is probable, because the waggling end segment of the chain is more disordered than the core.

Order parameters are evaluated both in nematic and smectic phases using isotropic internal field model of Vuks [14]. The variation of order parameter with temperature is shown in Fig. 2. As expected, it is interesting to note that the order parameter values are higher in the smectic phase than in the nematic state. This may be due to the arrangement of molecules of the smectic phase to be rigid and arranged perfectly parallel to one another in each layer.

The variation of the order parameter with the number of carbon atoms at a particular reduced temperature $\left(\left(T-T_{\mathrm{c}}\right) / T_{\mathrm{c}} \approx-0.024\right)$ is shown in Fig. 3 . Interestingly, odd even effect is observed in the order parameter as a function of $n$. The even number carbon atoms have higher order parameter when compared with odd number.

\section{Conclusion}

The reasonably good agreement found in polarizabilities, polarizability anisotropies and diamagnetic susceptibilities evaluated by the present method with the reported data, confirms the applicability of the molecular vibration approach to the cinnamate liquid crystals.

\section{References}

[1] J. Fripiat, Bull. Class. Sci. Acad. Rey. Belgrade 31, 39 (1945).

[2] R.J.W. Lefevre, D.A.A.S. Narayana Rao, Aust. J.Chem. 8, 39 (1955).

[3] B.P. Rao, V.R. Murthy, Ind. Chem. J. XIII, 17 (1979).

[4] C.G. Lefevre, R.J.W. Lefevre, D.A.A.S. Narayana Rao, J. Chem. Soc. 142, 708 (1956).

[5] W. Gordy, J. Chem. Phys. 14, 305 (1946).

[6] V.R. Murthy, Ind. J. Bio-Chem. Bio-Phys. 16, 43 (1979).

[7] V.R. Murthy, D.V. Subbaiah, S.V. Naidu, Ind. J. Bio. Chem. Bio. Phys. 16, 32 (1979).

[8] D.V. Subbaiah, M.S. Sastry, V.R. Murthy, J. Mol. Struct. 87, 105 (1981).

[9] D.V. Subbaiah, M.S. Sastry, V.R. Murthy, J. Phys. Chem. 87, 1730 (1983).

[10] D.V. Subbaiah, N. Sivaramaiah, V.R. Murthy, J. Polym. Mater. 9, 75 (1992).

[11] R.N.V. Ranga Reddy, S.V. Subrahmanayam, Mol. Cryst. Liq. Cryst. 89, 9 (1982).

[12] L. Pauling, The Nature of Chemical Bond, Oxford and IBII, New Delhi 1969.

[13] W. Maier, A. Saupe, Z. Nat.forsch. A 14, 882 (1959).

[14] M.F. Vuks, Opt. Spectrosc. 20, 361 (1966).

[15] H.E.J. Neugebauer, Can. J. Phys. 28, 292 (1950).

[16] Landolt-Börnstein Tabellen, Molekulen Teil 1, Springer Verlag, Berlin 1961.

[17] G. Pelzl, H. Sackmann, Trans. Faraday Soc. 5, 68 (1971).

[18] D. Krishnamurti, R. Somasekhar, Mol. Cryst. Liq. Cryst. 75, 133 (1981).

[19] P. Pascal, Ann. Chem. Phys. 5,19 (1910). 
[20] A. Pacault, Rev. Sci. 38, 86 (1948).

[21] W. Haberditzl, in: The Theory and Applications of Magnetic Susceptibility, Eds. L.N. Mulay, B.A. Boudreux, Wiley, New York 1976, Ch. 3.

[22] W. Haberditzl, Angew. Chem. Int. Ed. Engl. 5, 288 (1966).

[23] R.N.V. Ranga Reddy, V.R. Murthy, Y. Narasimha Murthy, K.V. Siva Kumar, Acta. Phys. Slov. 43, 375 (1993). 